\title{
Fabrication of One-Dimensional Photonic Crystals by Photoelectrochemical Etching of Silicon
}

\author{
Yu. A. Zharova ${ }^{a \wedge}$, G. V. Fedulova ${ }^{a}$, E. V. Gushchina ${ }^{a}$, A. V. Ankudinov ${ }^{a}$, \\ E. V. Astrova ${ }^{a}$, V. A. Ermakov ${ }^{b, c}$, and T. S. Perova ${ }^{b}$ \\ ${ }^{a}$ Ioffe Physical Technical Institute, Russian Academy of Sciences, St. Petersburg, 194021 Russia \\ ^e-mail:piliouguina@mail.ioffe.ru \\ ${ }^{b}$ Department of Electronic and Electrical Engineering, University of Dublin, Trinity College, Dublin, 2 Ireland \\ ${ }^{c}$ St. Petersburg State University of Information Technologies, Mechanics, and Optics, St. Petersburg, 197101 Russia \\ Submitted December 14, 2009; accepted for publication December 21, 2009
}

\begin{abstract}
The conditions of formation of deep periodic trenches by photoelectrochemical etching of $n$-Si (100) with linear seeds on the surface are analyzed. Criteria for the proper choice of the period of seed grooves and the etching current density in relation to the doping level of the substrate are formulated. Corrugation of walls is a characteristic feature of the obtained structures; this corrugation is caused by traces of merged macropores. Atomic-force microscopy is used to study roughness of the side-walls in relation to the etching conditions; the current density at which one can obtain the smoothest side-walls is determined. The roughness of the side walls in structures with periods of 7 and $9 \mu \mathrm{m}$ on Si with the resistivity of $15 \Omega \mathrm{cm}$ amounts to $\sim 40 \mathrm{~nm}$. It is shown that additional treatment of the structures in alkaline solutions can decrease the side-wall roughness by approximately a factor of 2 .
\end{abstract}

DOI: $10.1134 / \mathrm{S} 1063782610070213$

\section{INTRODUCTION}

Silicon-based periodic structures with deep trenches are widely used in various devices of integrated optics and photonics. These structures can be used as waveguide arrays, as an efficient anisotropic medium, as 1D photonic crystals, and as waveguides for controlling light within an integrated circuit. Previously, $1 \mathrm{D}$ photonic crystals with vertical walls and a large aspect ratio between the depth and width of the trench were fabricated by the method of anisotropic etching of silicon wafers with orientation (110) [1]. Studies of optical characteristics of such photonic crystals showed that they can be successfully used in the mid-IR region of the spectrum [2,3]. Later, similar structures were obtained using photoelectrochemical etching (PECE) of silicon with (100) orientation [4-7]; previously, this method had been mainly used to obtain a $2 \mathrm{D}$ periodic lattice in macroporous silicon [8-10]. The advantages of the PECE method as compared to anisotropic etching consist in the use of a substrate with the standard (100) orientation, in the absence of a necessity to precisely orient the pattern of the photomask with respect to crystallographic axes, and the possibility of fabricating simultaneously the trenches and macropores in a unified process $[11,12]$. However, the quality of the walls of the structures obtained by the PECE method is conventionally lower that in the case of trench-containing structures obtained by anisotropic etching; in the latter case, sil- icon edges are formed by optically smooth crystallographic planes (111).

The aim of this study was to gain insight into the conditions of etching and subsequent treatment, which make it possible to use the PECE method to obtain 1D structures with smooth walls.

\section{MAIN SYSTEMATIC FEATURES OF FORMATION OF 1D STRUCTURES}

In Fig. 1, we show the results of electron-microscopy study of a trench structure fabricated using the photoelectrochemical etching of $n$-Si (100) with the resistivity $\rho \approx 15 \Omega \mathrm{cm}$; linear nucleation centers in the form of seeding grooves were formed on the surface of this structure (see the inset in Fig. 1a).

The deep trenches shown in Fig. 1 were formed as a result of the coalescence of pores the traces of which give rise to corrugation of walls' surfaces (see also Fig. 2). The magnitude of roughness of the walls is an important parameter that controls optical characteristics of a 1D photonic crystal, since irregularities violate the coherence of optical waves reflected from the walls. This is especially evident in the case of reflection waves with short wavelengths, in which case the roughness of the surface is found to be comparable to the wavelength of the optical wave $[13,14]$. Formation of trenches from discrete macropores can be illustrated by the scheme shown in Fig. 3. As the normalized 

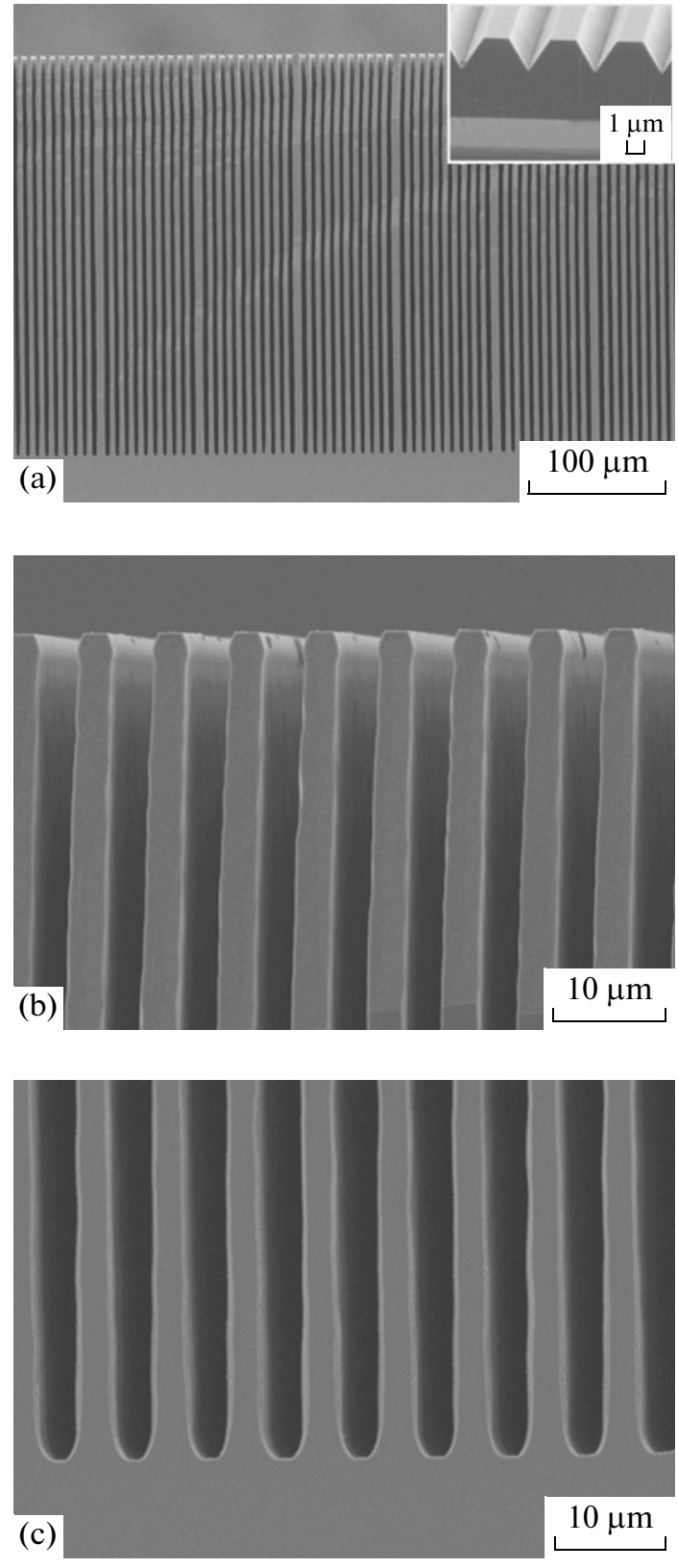

Fig. 1. An image (in a scanning electron microscope) of a $1 \mathrm{D}$ structure with the period $a=7 \mu \mathrm{m}$; the structure was obtained by the PECE method at relative current density $j / j_{\text {ps }}=0.41$ : panel (a) shows the image of the cross section (the inset shows the image of seeding grooves on the surface of the sample), panel (b) shows an enlarged image of the upper part of the structure, and panel (c) shows an enlarged image of the lower part of the structure.

etching-current density $j / j_{\mathrm{ps}}$ is increased, the distance between discreet pores decreases along the lines of seeds $A_{\|}$with subsequent coalescence of these pores $\left(j_{\mathrm{ps}}\right.$ is the critical current density corresponding to the transition from the bivalent solution of silicon to tetravalent one; i.e., to transition from pore formation to electrical polishing $[8,9])$. (a)

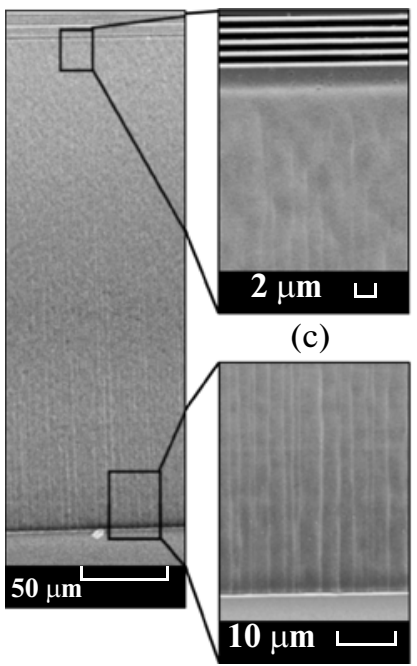

(d)

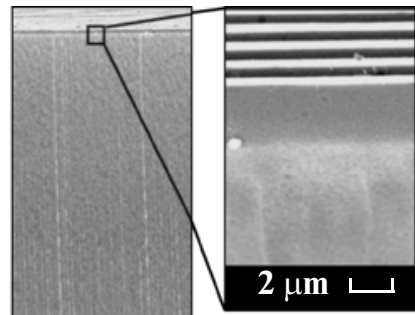

(f)

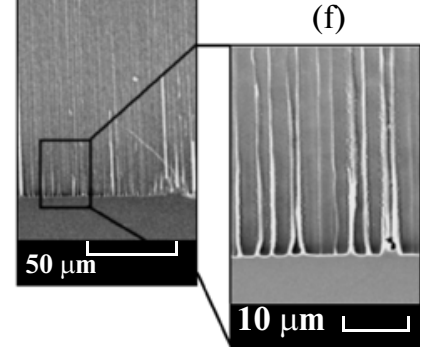

Fig. 2. Images (in a scanning electron microscope) of walls of the structure obtained by PECE at the current density $j / j_{\mathrm{ps}}=0.41$ : (a) for a structure with the period of $7 \mu \mathrm{m}$, (b) and (c) enlarged images of structure's upper and lower parts of the wall, and (d) the same for the structure with the period of $9 \mu \mathrm{m}$; (e) and (f) are enlarged images of the upper and lower parts of wall for this structure.

It has been established $[15,16]$ that the diameters of macropores in a semi-regular lattice is a constant quantity that is independent of either current or the period of seeding grooves $d \neq f(j, a)$. Thus, a siliconelectrolyte system tends toward the formation of macropores with a certain diameter $d$ characteristic of silicon with a specified resistivity. The low boundary for coalescence of pores corresponds to the condition that the mean distance between pores along the row $A_{\|}=d$, while the upper boundary corresponds to the trench width, which exceeds the macropores' diameter $w=2 d$ by twofold; the latter condition corresponds to the situation in which a trench is formed from a double row of pores. The main rules for obtaining the trench structures amount to the following two conditions [7]:

$$
\begin{gathered}
a \approx \bar{a}, \\
\frac{\pi d}{4 a}<\frac{j}{j_{\mathrm{ps}}}<\frac{2 d}{a},
\end{gathered}
$$

where $a$ is the period of seeding grooves and $\bar{a}$ is the average distance between macropores in the self-organizing lattice (the result of etching of a sample without seeds on the surface). In order to determine $\bar{a}$, we can use the mnemonic rule $\bar{a} \approx 2 \sqrt{\rho}$. Thus, to obtain a high-quality trench structure, one has, first of all, to reconcile the distance between seeding grooves with the level of silicon doping. The cause of large roughness of the walls may be either incomplete coalescence of macropores (Fig. 4a) or the effect of twinning, i.e., 


\begin{tabular}{|c|c|c|}
\hline 1 & $\begin{array}{c}\text { Discrete pores } A_{\|} \gg d \\
\quad j / j_{\mathrm{ps}} \ll \pi d / 4 a\end{array}$ & $\bullet \rightarrow \frac{1}{A_{\|}}$ \\
\hline 2 & $\begin{array}{c}\text { Discrete pores } A_{\|}>d \\
j / j_{\mathrm{ps}}<\pi d / 4 a\end{array}$ & 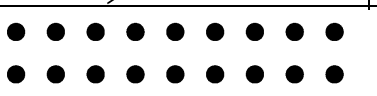 \\
\hline 3 & $\begin{array}{c}\text { Coalescence } \\
\text { threshold } A_{\|}>d \\
j / j_{\mathrm{ps}}=\pi d / 4 a\end{array}$ & 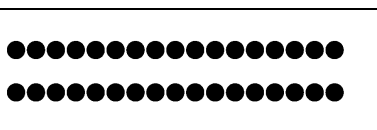 \\
\hline 4 & $\begin{array}{c}\text { Formation of continuous } \\
\text { trenches } A_{\|}<d \\
j / j_{\mathrm{ps}}>\pi d / 4 a\end{array}$ & \\
\hline 5 & $\begin{array}{l}\text { Widening of the trenches } \\
\text { and wall smoothening } \\
\qquad a \approx \bar{a} \\
\qquad \pi d / 4 a<j / j_{\mathrm{ps}}<2 d / a\end{array}$ & \\
\hline 6 & $\begin{array}{c}\text { Formation of double } \\
\text { rows of pores } \\
w>2 d \\
j / j_{\mathrm{ps}}>2 d / a\end{array}$ & \\
\hline
\end{tabular}

Fig. 3. A schematic diagram elucidating conditions of coalescence of macropores with formation of periodic trench structures, an idealized pattern disregarding the random distribution of pores along the rows. Here, $d$ is the diameter of a micropore, $a$ is the period of seeding lines, $A_{\|}$is the distance between pores along the seeding lines, and $j / j_{\mathrm{ps}}$ is the relative current density.

formation of trenches of a double row of macropores (Fig. 4b). Consequently, a trench structure with relatively smooth walls is formed only in a certain range of current densities (2).

In Fig. 5, we show the dependences $j / j_{\mathrm{ps}}=f(a)$ for the upper and lower boundaries of the etching current at which the trench structure is formed [7]. The graypainted region between these boundaries is defined by inequality (2). The middle of range (2) corresponds to the relative current density

$$
\frac{j}{j_{\mathrm{ps}}} \approx \frac{1.4 d}{a}
$$

and only approximately indicates which current density should be used in etching. To determine the optimal value of current at which roughness of the walls is at a minimum, we conducted studies based on the use of atomic-force microscopy (AFM) and scanning electron microscopy (SEM).

\section{A STUDY OF THE ROUGHNESS OF WALLS IN RELATION TO THE CURRENT DENSITY DURING ETCHING}

The initial material in the experiment was $n$-Si (100) with the resistivity $\rho=15 \Omega \mathrm{cm}$; for this material, $\bar{a}=$ $8 \mu \mathrm{m}$ and $d=3 \mu \mathrm{m}$. A good ohmic contact for photoelectrochemical etching was provided by ion-implan-
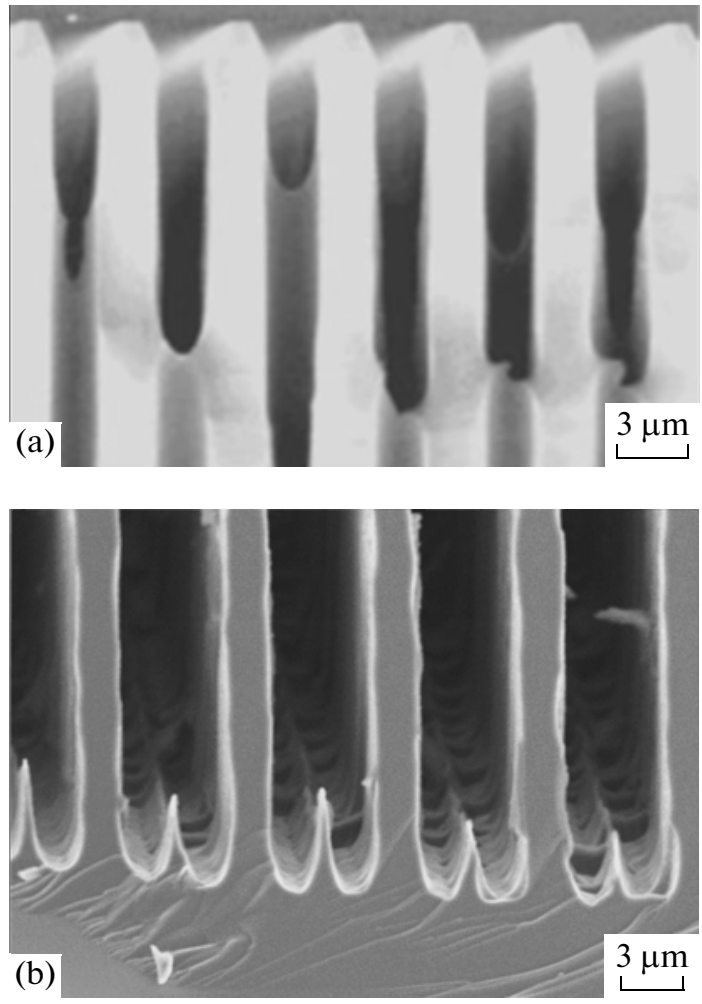

Fig. 4. An image obtained in a scanning electron microscope of a cross section of structures (a) with incompletely coalesced pores and (b) with bifurcation of the bottom of trenches (the twinning effect).

tation doping with phosphorus of the rear side of the silicon wafer. A plasmochemical oxide in which narrow and long windows oriented along the direction $\langle 110\rangle$ were formed using a photomask was used as a mask for formation of the pattern of seeding grooves. At the sample surface, there were four zones with different period of seeding grooves $a=7-13 \mu \mathrm{m}$ (for details, see [7]). For studies, we chose structures with periods $a=7$ and $9 \mu \mathrm{m}$, which are close to the average value of $\bar{a}$ for self-organizing macropores in the PECE array; the structures were studied employing an ET and TE setup and using a water-alcohol solution containing $4 \%$ of hydrofluoric acid, at a temperature $T=$ $20^{\circ} \mathrm{C}$, and at the voltage at the $\mathrm{Si}-$ electrolyte interface equal to $1-2 \mathrm{~V}$. The depth of etching was $200-300 \mu \mathrm{m}$. In order to compensate for the effect related to a decrease in the HF concentration in the depth part trenches, we used a correction of the current density in time, which ensured the fulfillment of the condition $j / j_{\mathrm{ps}}=$ const [8]. Such correction is efficient in the case of discrete macropores, keeping their diameter constant over the depth, but does not ensure the equal roughness of the walls in the trench structures. As a rule, the surface of the upper part of the wall was found to be smoother, while vertical grooves are observed in the relief of the lower part (see, for example, Fig. 2). 
Prior to experimental studies, the obtained structures were cleaved along trenches; typically, a portion of the wall at a depth of $120 \mu \mathrm{m}$ from the upper surface of the sample was chosen. Obtained cleavages were studied using an OPTEM optical microscope with a Mitutoyo objective lens, making it possible to attain a resolution of $1 \mu \mathrm{m}$ in the plane, and using an Integra Aura atomic-force microscope produced by the NTMDTCo. (Russia). The AFM studies were conducted under resonance conditions with an average force of interaction of about $1 \mathrm{nN}$. We used NSG01 and HA-NC commercial probes with a radius of tip curvature no larger than $30 \mathrm{~nm}$ in both cases, with of characteristic resonance frequencies 150 and $120 \mathrm{kHz}$, and with force constants 5 and $3.5 \mathrm{~N} / \mathrm{m}$, respectively.

In Fig. 6, we show the topography of the relief of the walls formed at different current densities of PECE. The arrays of data forming the image were processed with a gradient filter, which made it possible to clearly reveal the fine features of the relief. Vertical grooves observed in all three images are the traces of macropores and are oriented along the crystallographic direction $\langle 100\rangle$. As the relative current density of etching is increased, the height of irregularities varies nonmonotonically. At small values of $j / j_{\mathrm{ps}}$ (Fig. 6a), the large roughness of the wall is related to incomplete coalescence of pores and, at large values of $j / j_{\mathrm{ps}}$, the large roughness is apparently related to the initial stage of twinning.

The results of the AFM study of wall surfaces for structures that had periods of 7 and $9 \mu \mathrm{m}$ and were fabricated in a wide range of current densities are shown in Fig. 7.

It can be seen from Fig. 7 that the dependence RMS $=f\left(j / j_{\mathrm{ps}}\right)$ is nonmonotonic: the smoothest walls with the smallest $\mathrm{RMS} \approx 40 \mathrm{~nm}$ are formed in the range of current densities $0.34<j / j_{\mathrm{ps}}<0.44$. The roughness sharply increases on both sides of this range. Thus, the optimal value of the current density corresponds to $j / j_{\mathrm{ps}}=0.39$, which is smaller than the value $j / j_{\mathrm{ps}}=0.525$ calculated using formula (3). Taking into account that $j_{\mathrm{ps}}=31 \mathrm{~mA} / \mathrm{cm}^{2}$, we obtain $j=$ $12 \mathrm{~mA} / \mathrm{cm}^{2}$ as a result of our measurements; the latter value is twice as large as the current density $6 \mathrm{~mA} / \mathrm{cm}^{2}$ optimized for etching of discrete macropores in silicon with the same electrical conductivity [17]. It is worth noting that the roughness of the walls is extremely sensitive to even small changes in parameters of the structure. For example, in an SEM image of the walls for structures with different periods (Fig. 2) etched in a single process at the current density amounting to $j / j_{\mathrm{ps}}=0.41$ close to the optimal value, we can clearly see the difference between the surface morphologies. Structures with the period $7 \mu \mathrm{m}$ feature smoother walls than do structures with the period $9 \mu \mathrm{m}$, in spite of the fact that condition (1) is satisfied in both cases.

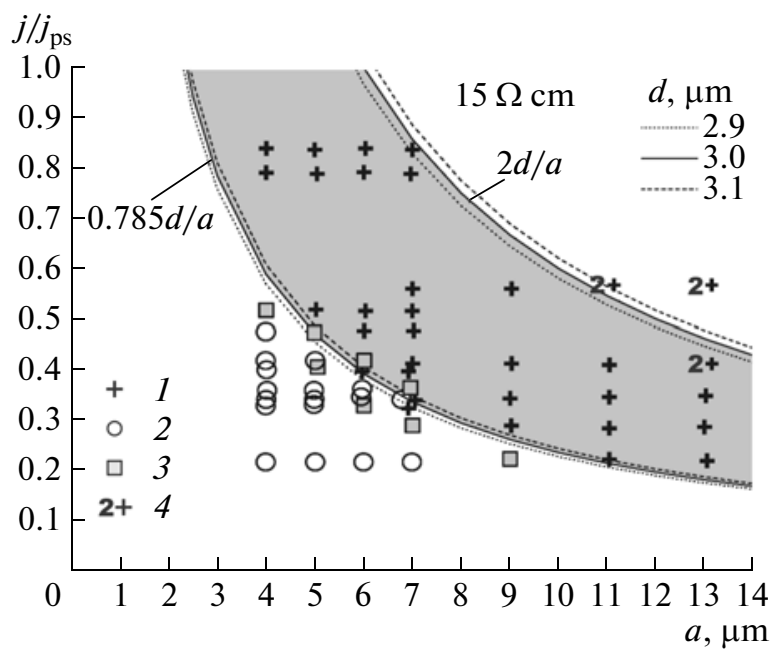

Fig. 5. Dependences of current required for coalescence of discrete pores with formation of trenches and for appearance of double pores on the period of seeding grooves for silicon with the resistivity $15 \Omega \mathrm{cm}$. The lines correspond to calculated dependences $j / j_{\mathrm{ps}}=0.785 d / a$ and $j / j_{\mathrm{ps}}=$ $2 d / a$ for pore diameters $d=2.9,3.0$, and $3.1 \mu \mathrm{m}$. Graphic symbols represent experimental data for (1) pores coalesced into continuous trenches, (2) discrete pores, (3) the intermediate case of incompletely coalesced pores, and (4) trenches formed of a double row of pores.

\section{ADDITIONAL TREATMENT OF TRENCH STRUCTURES IN ALKALINE SOLUTIONS}

To smooth further the walls' surface of the structures formed in the course of PECE, we studied the methods of alkaline etching. The side surfaces of the trench structures are formed by $\{110\}$ planes. In order to smooth the relief of these surfaces, one should use an alkaline anisotropic etchant, which etches the (110) planes with a rate lower than that for etching of other crystallographic planes. Typically, an alkaline solution etches the $\mathrm{Si}$ (111) crystal plane with the lowest rate, while a ratio between the etching rates of the (100) and (110) planes can be controlled [18, 19]. Conventionally, solutions with a high concentration of $\mathrm{KOH}$ are used for etching of Si; etching is carried out at high temperatures, in which case the etching rate $R_{\{100\}}<R_{\{110\}}$. This relation can be reversed by lowering the temperature of the process in combination with the addition of isopropyl alcohol or its isomer propanol [20]. As was shown previously [18], such additives make it possible to smooth silicon surfaces formed by different crystallographic planes. Nilsson et al. [21] managed to reduce the roughness of vertical walls formed by (110) planes in structures obtained by the method of dry reactive-ion etching (DRIE), from 70 to $30 \mathrm{~nm}$, using treatment of structure's walls in an aqueous solution containing $3 \mathrm{M}$ of $\mathrm{KOH}$ and $2 \mathrm{M}$ of isopropyl alcohol.

Our experiments with smoothing of the walls were carried out using structures with the period of $7 \mu \mathrm{m}$ 

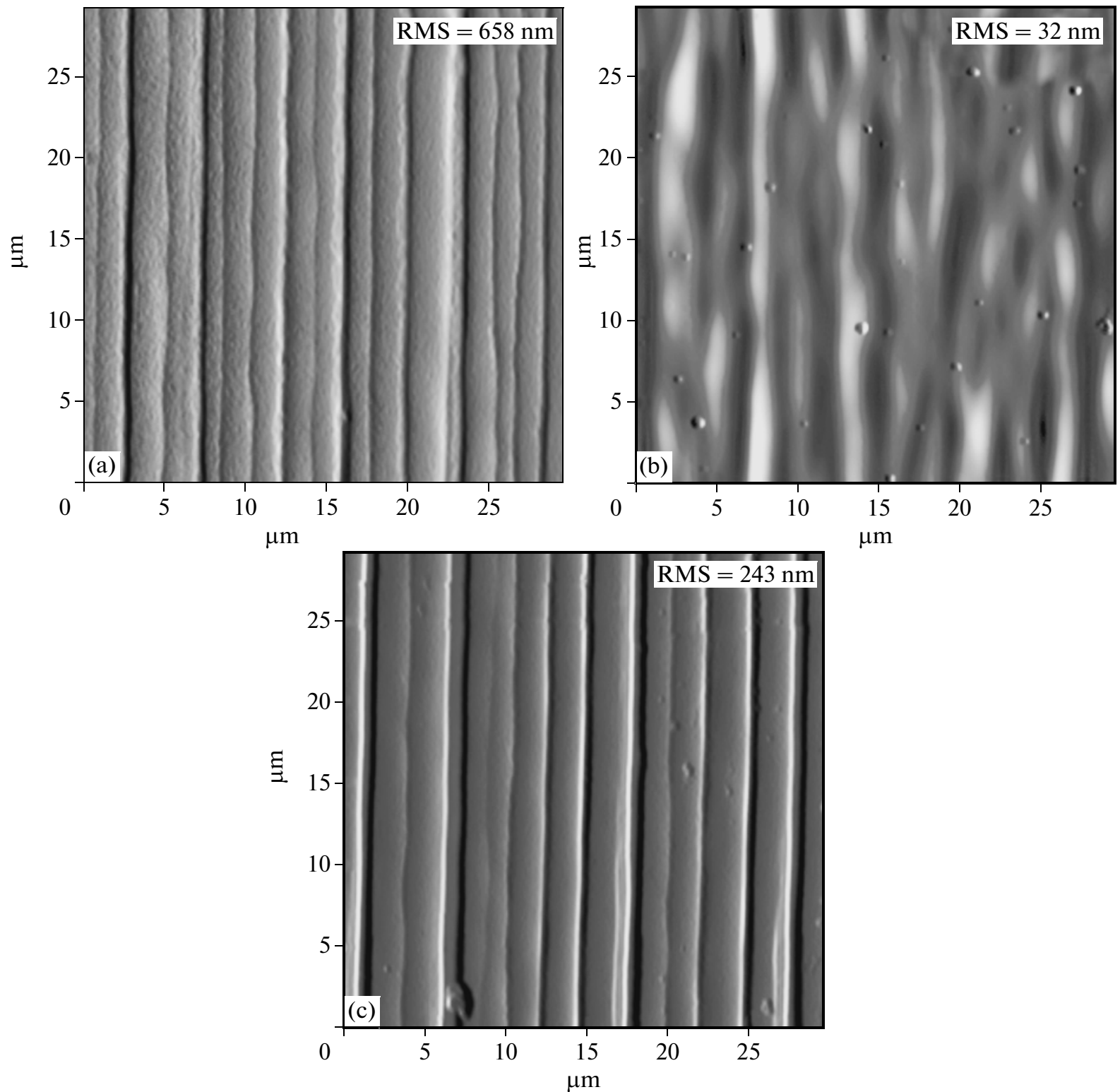

Fig. 6. AFM images of walls' surfaces for structures with the period $a=7 \mu \mathrm{m}$; the structures were obtained at relative etchingcurrent densities $j / j_{\mathrm{ps}}=$ (a) 0.22 , (b) 0.4 , and (c) 0.48 .

and with through trenches for uniform penetration of the solution over the entire depth. For treatment, we used an aqueous solution of $1-5 \% \mathrm{KOH}$ to which propanol was added with the volume ratio $1: 2$. The sam-

Table 1. Roughness of walls in structures treated in solutions containing $\mathrm{KOH}$

\begin{tabular}{c|c|c}
\hline Fig. 8 & KOH concentration, \% & $\mathrm{nm}$ \\
\hline$a$ & Initial sample & 45 \\
$b$ & 1.4 & 29 \\
$c$ & 2.7 & 23 \\
$d$ & 5.2 & 44 \\
\hline
\end{tabular}

ples were treated for $1 \mathrm{~h}$ at a temperature of $14^{\circ} \mathrm{C}$. The results of AFM studies of the samples treated in solutions with different concentrations of alkali are shown in Fig. 8. It can be seen that vertical grooves on the wall of the initial structure completely disappear as a result of treatment; instead, grooves with shorter periods of disposition in the horizontal direction appear. The values of RMS are listed in Table 1.

A solution containing $2.7 \%$ of $\mathrm{KOH}$ was chosen for studies of variations in the roughness of the walls with the etching time. The experiments were carried out using the structures with the period of $9 \mu \mathrm{m}$, fabricated at the current density $j / j_{\mathrm{ps}}=0.35$. The initial sample featured a predominantly vertical corrugation, which 


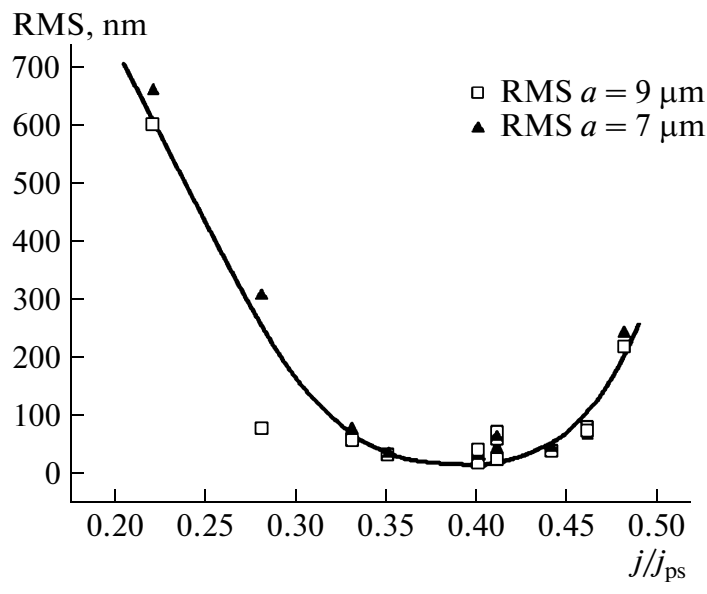

Fig. 7. The root-mean-square roughness of walls in structures with periods 7 and $9 \mu \mathrm{m}$ as a function of the normalized PECE current density $j / j_{\mathrm{ps}}$. coincided with the direction of growth of the pores (Table 2). In the case of short durations of the chemical treatment, vertical grooves disappear, which brings about a decrease in the roughness of the surface; however, transverse corrugation in the $\langle 110\rangle$ direction appears with the amplitude increasing as duration of the treatment is increased. Formation of the etch patterns elongated along the $\langle 110\rangle$ direction is conventionally observed in the case of treatment of polished surface of the (110) wafers in an alkaline solution [22]. It can be seen from the data in Table 2 that the optimal duration of treatment was about $1 \mathrm{~h}$. This duration is sufficient for smoothing the initial corrugation; at the same time, the duration of the process is not sufficiently long for development of a deep relief oriented in the horizontal direction. The important conclusion here amounts to the statement that there are parame-
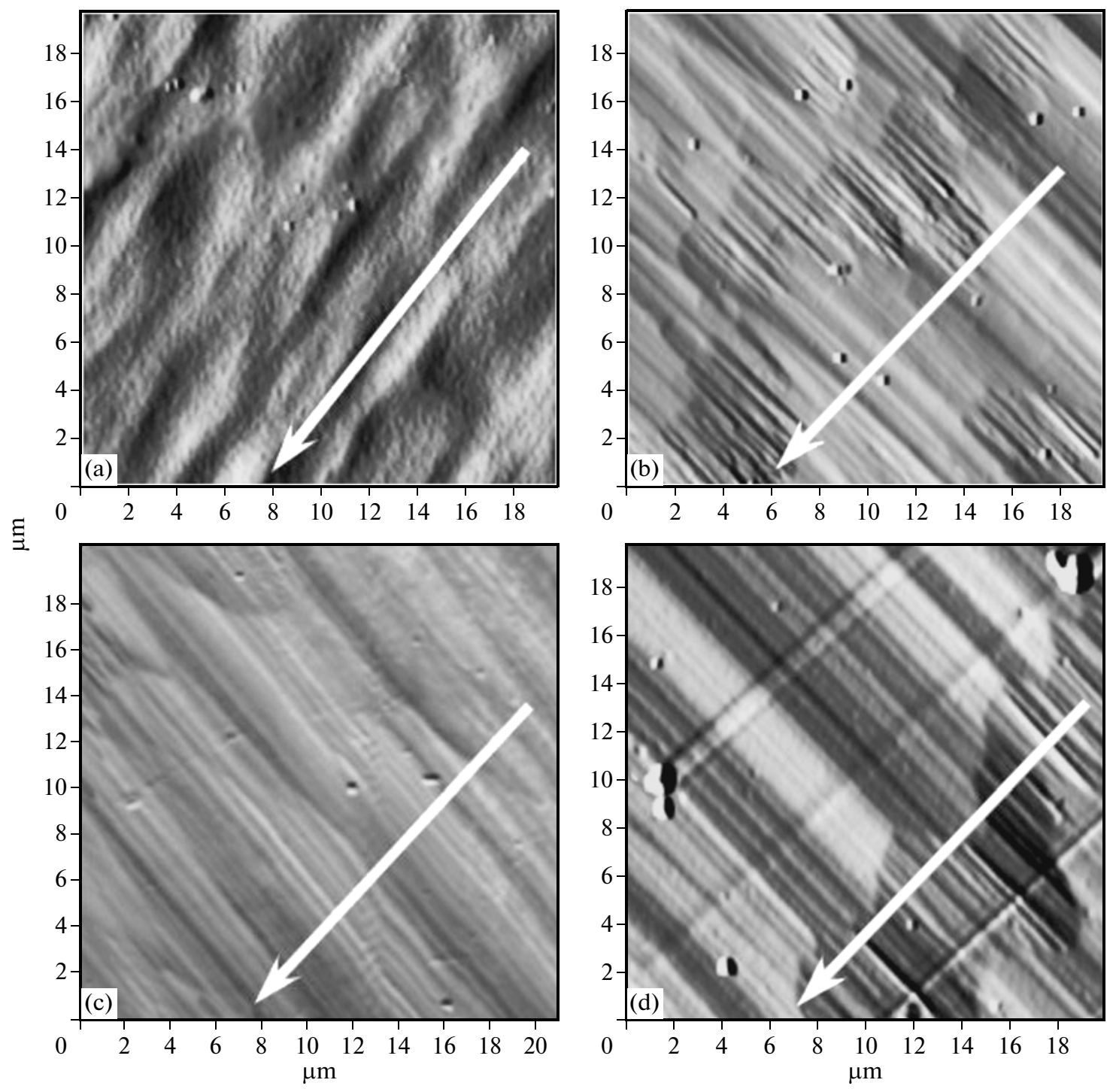

Fig. 8. The AFM images illustrating the transformation of the relief of a silicon wall in (a) the as-prepared sample and after chemical treatment of the sample for $1 \mathrm{~h}$ at $T=14^{\circ} \mathrm{C}$ in a solution with $\mathrm{KOH}$ concentration (b) 1.4 , (c) 2.7 , and (d) $5.2 \%$. The arrows indicate the direction of vertical grooves in the as-prepared sample. 
Table 2. Images (in the optical and electron microscopes) of walls of the structure treated at $14^{\circ} \mathrm{C}$ in a solution containing $2.7 \% \mathrm{KOH}$ for various durations of treatment

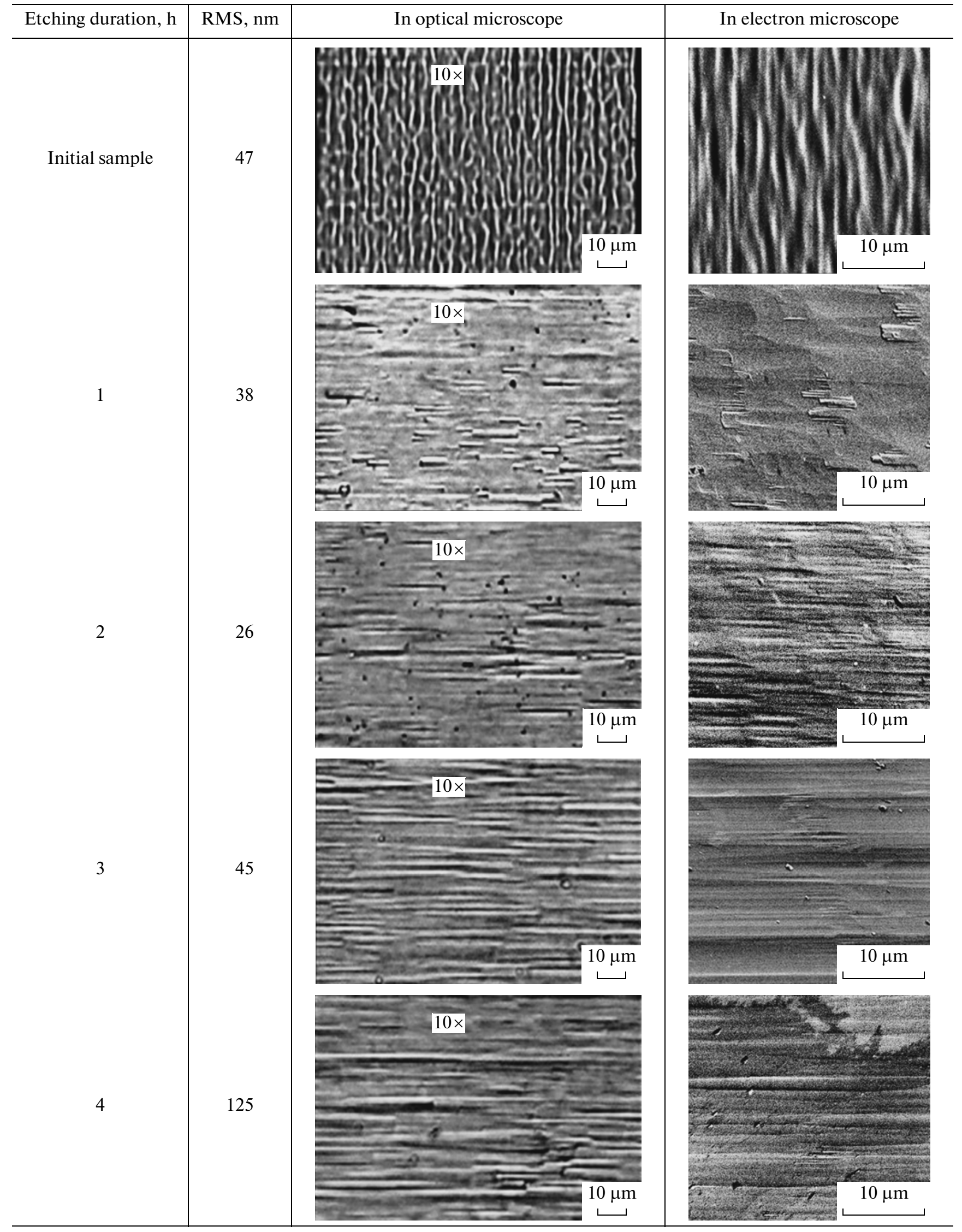


ters of additional treatment of trench structures in alkaline solutions such that they can be used to smooth the roughness of the walls by approximately a factor of 2 .

\section{CONCLUSIONS}

Thus, it is established that the range of current densities in etching at which smooth walls are obtained in trench structures is narrower than that following from criterion (2) reflecting only the condition for coalescence of pores into a unified trench and the absence of twinning. The roughness of walls in the structures obtained by the PECE method can be decreased using additional treatment in an alkaline solution with addition of propanol; however, the obtained roughness of the walls at a level of $\sim 25 \mathrm{~nm}$ is still higher than that in the trench structures obtained by the method of anisotropic etching of silicon (110) (RMS $\leq 6 \mathrm{~nm})$.

\section{ACKNOWLEDGMENTS}

This study was supported by the Russian Foundation for Basic Research (projects nos. 08-02-01408 and 09-02-00782), a Scientific School grant of the President of the Russian Federation (no. 2951.2008-2), State Contract FANI GK no. 02.740.11.05, the programs of the Presidium of the Russian Academy of Sciences "Basics of Fundamental Studies of Nanotechnologies and Nanomaterials" and "Quantum Physics of Condensed Materials," and an IRCSET grant (Ireland).

\section{REFERENCES}

1. E. V. Astrova, T. S. Perova, V. A. Tolmachev, A. D. Remenyuk, J. Vij, and A. Moore, Fiz. Tekh. Poluprovodn. 37, 417 (2003) [Semiconductors 37, 399 (2003)].

2. V. Tolmachev, E. Astrova, T. Perova, J. Pilyugina, and A. Moore, Phys. Stat. Solidi C 2, 3288 (2005).

3. V. A. Tolmachev, E. V. Astrova, J. A. Pilyugina, T. S. Perova, A. Moore, and J. K. Vij, Opt. Mater. 27, 831 (2005).

4. G. Barillaro, A. Nannini, and F. Pieri, J. Electrochem. Soc. 149, 180 (2002).
5. G. Barillaro, A. Nannini, and M. Piotto, Sens. Actuat. A 102, 195 (2002).

6. E. V. Astrova, A. A. Nechitailov, V. A. Tolmachev, V. A. Melnikov, and T. S. Perova, Phys. Stat. Solidi A 206, 1235 (2009).

7. E. V. Astrova and G. V. Fedulova, J. Micromech. Microeng. 19, 095009 (2009); http://stacks.iop.org/JMM/ $19 / 000000$

8. V. Lehmann and H. Foll, J. Electrochem. Soc. 137, 653 (1990).

9. V. Lehmann, Electrochemistry of Silicon (Wiley-VCH, 2002).

10. H. Foll, M. Christophersen, J. Carstensen, and G. Hasse, Mater. Sci. Eng. R 39, 93 (2002).

11. T. Geppert, S. L. Schweizer, U. Gosele, and R. B. Wehrspohn, Appl. Phys. A 84, 237 (2006).

12. R. B. Wehrspohn, S. L. Schweizer, and V. Sandoghdar, Phys. Stat. Solidi A 204, 3708 (2007).

13. E. V. Astrova, V. A. Tolmachev, G. V. Fedulova, V. A. Melnikov, A. V. Ankudinov, and T. S. Perova, in Appl. Phys. A, DOI 10.1007/s00339-009-5469-7.

14. E. V. Astrova, V. A. Tolmachev, G. V. Fedulova, V. A. Melnikov, and T. S. Perova, Izv. RAN, Ser. Fiz. 74, 72 (2010).

15. E. V. Astrova and A. A. Nechitailov, Fiz. Tekh. Poluprovodn. 42, 747 (2008) [Semiconductors 42, 746 (2008)].

16. E. V. Astrova, A. A. Nechitailov, V. A. Tolmachev, V. A. Melnikov, and T. S. Perova, Phys. Stat. Solidi A 206, 1235 (2009).

17. E. V. Astrova, T. N. Borovinskaya, T. S. Perova, and M. V. Zamoryanskaya, Fiz. Tekh. Poluprovodn. 38, 1121 (2004) [Semiconductors 38, 1088 (2004)].

18. A. Zubel and M. Kramkowska, Sens. Actuat. A 93, 138 (2001).

19. A. Zubel and M. Kramkowska, Sens. Actuat. A 101, 255 (2002).

20. V. Lehmann, Phys. Stat. Solidi A 204, 1318 (2007).

21. D. Nilsson, S. Jensen, and A. Menon, J. Micromech. Microeng. 13, S57 (2003).

22. E. van Veenendaal, K. Sato, M. Shikida, and J. van Suchtelen, Sens. Actuat. A 93, 219 (2001).

Translated by A. Spitsyn 